\title{
SHOUld I STAY OR SHOULD I GO? EVIDENCE ACCUMULATION DRIVES DECISION MAKING IN HUMAN DRIVERS
}

\author{
Arkady Zgonnikov \\ AiTech \& Department of Cognitive Robotics \\ Delft University of Technology \\ Mekelweg 2, 2628 CD Delft, Netherlands \\ a.zgonnikov@tudelft.nl
}

\author{
David Abbink \\ Department of Cognitive Robotics \\ Delft University of Technology \\ Mekelweg 2, 2628 CD Delft, Netherlands \\ d.a.abbink@tudelft.nl
}

\author{
Gustav Markkula \\ Institute for Transport Studies \\ University of Leeds \\ 34-40 University Road, Leeds LS2 9JT, UK \\ g.markkula@leeds.ac.uk
}

\begin{abstract}
Understanding decisions of human drivers is essential for the development of safe and efficient transportation systems. However, current models of decision-making in drivers provide little insight into the underlying cognitive processes. On the other hand, laboratory studies of abstract, highly controlled tasks point towards noisy evidence accumulation as a key mechanism governing decision making. Yet it is unclear whether the cognitive processes implicated in these tasks are as paramount to decisions that are ingrained in more complex behaviors, such as driving. Here we aim to address the gap between studies of naturalistic decision making in drivers and modern cognitive models of decision making. We investigate drivers' decision making during unprotected left turns in a simulated driving task, and model the cognitive process underlying these decisions. We show that the model explains the observed decision outcomes and response times, as well as substantial individual differences in those. Through cross-validation, we demonstrate that the model not only explains the data, but also generalizes to out-of-sample conditions, effectively providing a way to predict human drivers' behavior in real time. Our results reveal the cognitive mechanisms of gap acceptance decisions in human drivers, and exemplify how simple cognitive process models can help us to understand and predict human road user behavior.
\end{abstract}

\section{Introduction}

Understanding the seemingly mundane decisions made daily by millions of human drivers is critical for designing traffic infrastructure and engineering automated vehicles which can safely interact with humans around them. Overtaking a slow-moving vehicle, merging onto a highway, taking a left turn across path of an oncoming car — all these situations require the driver to make an informed and well-timed decision whether or not to accept a space- and time-gap. Gap acceptance models have been instrumental in clarifying which factors affect these decisions. These models, developed with support from both naturalistic data and driving simulator studies, typically describe what information drivers use, and predict what decisions they make (Davis \& Swenson, 2004 Farah, Bekhor, Polus, \& Toledo, 2009 Hamed, Easa, \& Batayneh, 1997. Toledo, 2007). Still, little is known about how the drivers process the relevant perceptual information while arriving to a decision. Understanding and modeling the underlying cognitive mechanisms can lead to more generalizable predictions of these decisions and help to predict how dynamic changes in the environment over the time course of a decision affect the behavior (Jarecki, Tan, \& Jenny, 2020). 
Laboratory studies of abstract, highly controlled tasks have pointed towards noisy evidence accumulation as the primary mechanism governing human decision making (Gold \& Shadlen, 2007, Ratcliff \& McKoon, 2008 Stine, Zylberberg, Ditterich, \& Shadlen, 2020). However, it is unclear whether the mechanisms implicated in simple perceptual decisions in the laboratory are as paramount to decisions that are part of complex behaviors like driving. In tasks such as gap acceptance, the available perceptual information is much richer, often varying continuously over time, and motor behaviors are more complex than in traditional laboratory-based decision-making tasks such as motion or orientation discrimination. Even carefully designed abstract tasks can deprive human subjects of the potentially relevant context, the senses of agency and embodiment (Matusz, Dikker, Huth, \& Perrodin, 2019 Shamay-Tsoory \& Mendelsohn, 2019). This could hinder understanding of the cognitive mechanisms of interest, and it is far from obvious how to generalize from simple laboratory paradigms on perceptual choice to driving situations. Recently, first steps have been taken toward applying evidence accumulation models in driving and road traffic contexts, but these have been limited to speeded responses to discrete stimuli (Ratcliff, 2015, Ratcliff \& Strayer, 2014), or have not been fully stringent in model analyses (Giles et al., 2019 Markkula, Boer, Romano, \& Merat, 2018; Xue, Markkula, Yan, \& Merat, 2018). Therefore, there is currently a lack of principled investigations of evidence accumulation in contexts where drivers need to consider more complex traffic situations where the correct response is not immediately clear from the stimulus.

The aim of this paper is to bridge the gap between studies of human road user behavior and models of decision making elaborated in basic cognitive science. To this end, we developed an experimental method that allowed drivers to make a decision that is very common in everyday driving - accepting or rejecting a gap when turning across oncoming traffic — but in a way that simultaneously permitted stringent modelling. Using this method, we measured how the participants' decisions and response times varied with distance and time-to-arrival of the oncoming vehicle. We developed a model to capture the underlying cognitive processes determining the gap acceptance decisions, representing the drivers' decision making as a dynamic process based on accumulation of noisy evidence over time. We then fitted the developed model to the individual participants' data. The model captured the between- and within-participant variability in decision outcomes and response times, and successfully predicted the observed behavior in out-of-sample conditions. The results suggest that evidence accumulation of distance and time-to-arrival under time pressure underlies drivers' decision making during left-turn gap acceptance, and illustrate how simple cognitive models can explain human road user behavior.

\section{Methods}

This research complied with the tenets of the Declaration of Helsinki and was approved by the Human Research Ethics Committee of TU Delft. Informed consent was obtained from each participant.

\section{Participants}

Sixteen participants (mean age 27 (range: 22 to 34 ) years old; 10 male, 6 female) performed a virtual driving task in exchange for a gift voucher worth $€ 15$. The participants had been in possession of a driving license for 8 (range: 3 to 18) years on average, of which they have been driving regularly (as interpreted by the participant) for the average of 5 (range: 0 to 18 ) years.

\section{Setup}

The participants performed the experiment in a first-person-view fixed-base driving simulator, which included a 65-inch screen and a commercially available Logitech G29 steering wheel (Figure 1). The distance between the center of the screen and the participants' eyes was approximately 1.5m. Carla (Dosovitskiy, Ros, Codevilla, Lopez, \& Koltun, 2017) was used as a simulation software.

\section{Protocol}

Each participant was asked to drive in a virtual urban area $(1.5$ by $1.5 \mathrm{~km}$, a regular grid of square blocks 150 by 150 meters each), following eight randomly generated routes. Each route included 25 intersections; the participants were instructed to go straight on 5 intersections, turn right on 5 intersections, and turn left on 15 intersections (the order of the turns was randomized). This resulted in 120 left turns per participant in total.

The instructions were provided to the participants via auditory navigation prompts which were repeated twice, 120 meters and 30 meters before the intersection. The participants were told that there will be traffic 


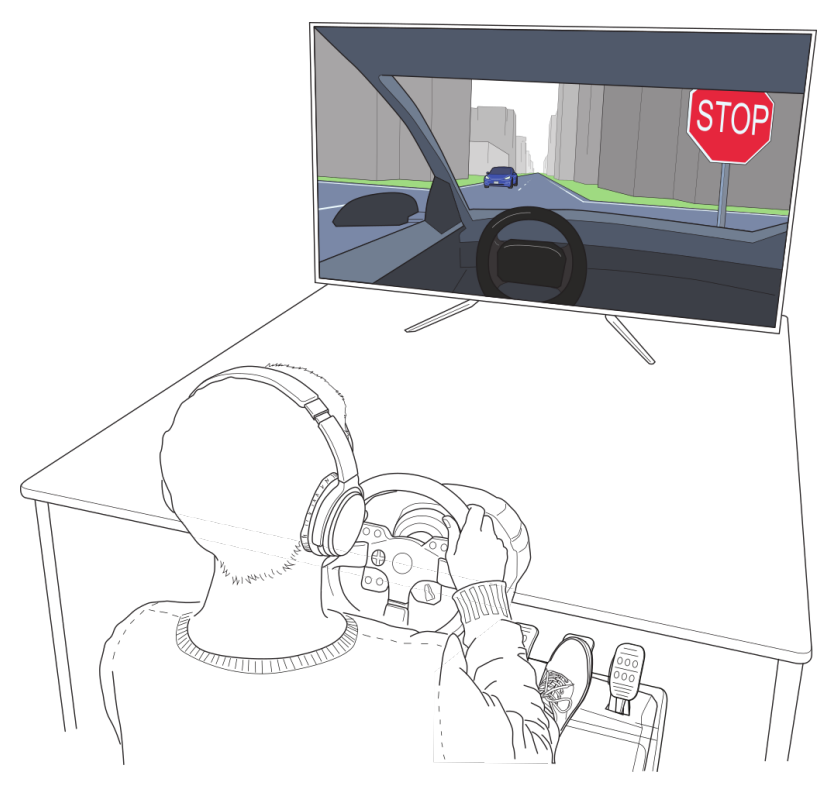

Figure 1: Experimental setup.

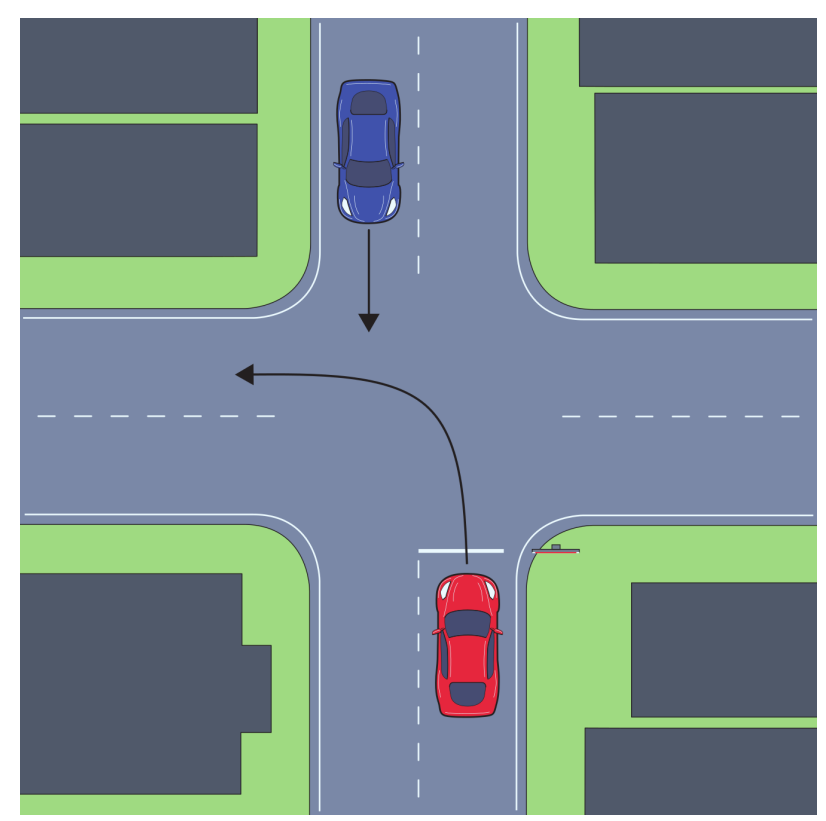

Figure 2: Top-down view of the left-turn interaction.

coming from the opposite direction, and were instructed to fully stop the vehicle at the stopping line on each intersection, which was also marked by the stop sign.

During left turns (Figure 2), when the driver stopped at the intersection, an oncoming vehicle instantaneously appeared at a randomly chosen distance $(90,120$, or $150 \mathrm{~m})$ across the intersection. The initial time-to-arrival (TTA, 4, 5, or $6 \mathrm{~s}$ ) of the oncoming vehicle was randomly chosen for each left turn. Time-to-arrival conditions were balanced within each route, such that on each route there were exactly five left turns at each of the three time-to-arrival levels. To summarize, we used a 3-by-3 factorial design, varying initial time-to-arrival and distance to the oncoming vehicle.

The speed of the oncoming vehicle at the moment of its appearance at the intersection was calculated as the ratio of initial distance to initial time-to-arrival (Table 1), and was held constant at that level throughout 


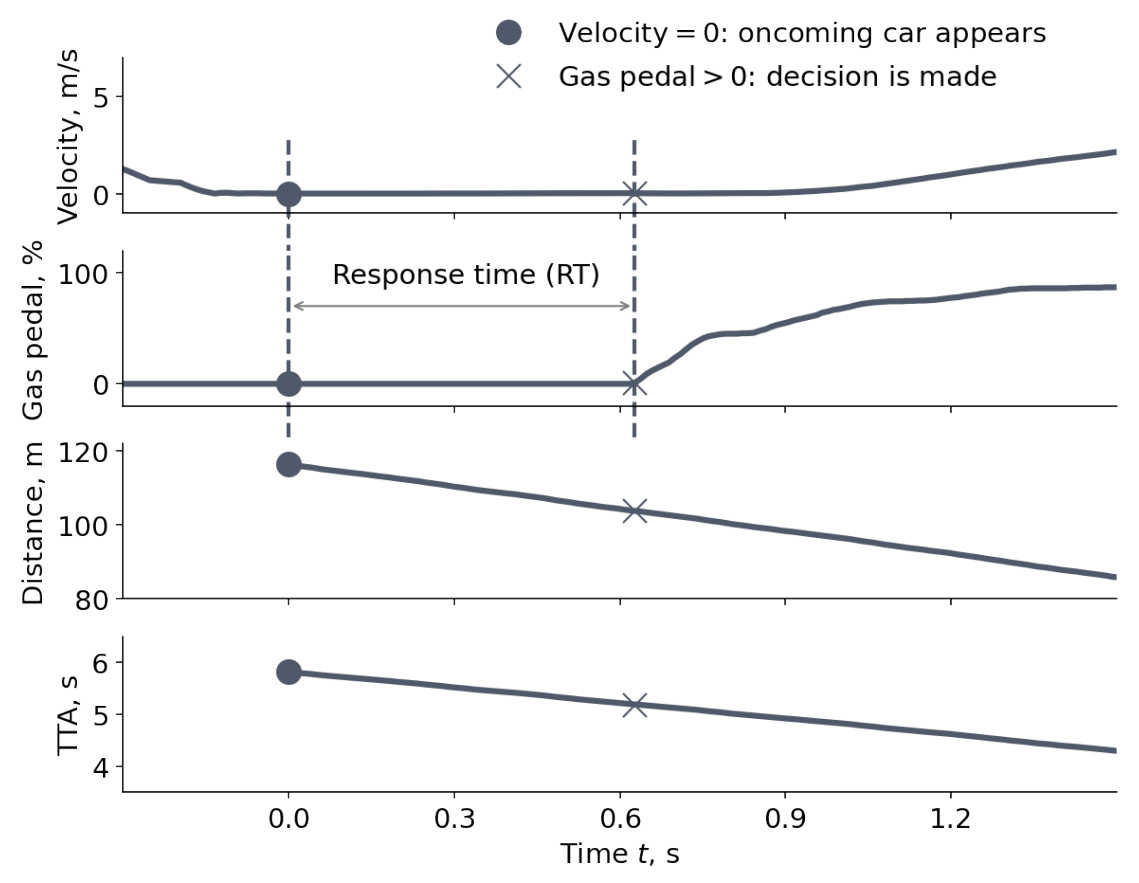

Figure 3: Time traces of a typical interaction, showing the participant decelerating for the stop sign, the oncoming car appearing at a constant speed at $t=0$, the dynamics of distance and time-to-arrival (TTA), and the moment when the participant presses the gas pedal to cross in front of the oncoming car.

the trial. The participants were required to decide whether to go before the oncoming car arrived, or stay, i.e. wait until the car passed the intersection and only then continue the route. Positions, velocities, and accelerations of the two vehicles during the interaction were recorded at $100 \mathrm{~Hz}$.

\begin{tabular}{llll}
\hline distance $\backslash$ TTA & $4 \mathrm{~s}$ & $5 \mathrm{~s}$ & $6 \mathrm{~s}$ \\
\hline $90 \mathrm{~m}$ & 22.5 & 18 & 15 \\
$120 \mathrm{~m}$ & 30 & 24 & 20 \\
$150 \mathrm{~m}$ & 37.5 & 30 & 25 \\
\hline
\end{tabular}

Table 1: Experimental conditions and the associated values of the speed $(\mathrm{m} / \mathrm{s})$ of the oncoming vehicle

\section{Data analysis}

Initial time-to-arrival and distance were the independent variables. Decision (go or stay) and response time (only for "go" decisions) were the dependent variables. The decision was determined based on whether the participant crossed the intersection before the oncoming vehicle. Response time was defined as the time between the appearance of the oncoming vehicle and the time when the participant first pressed the gas pedal after the oncoming vehicle appeared (Figure 3). For "turn" decisions, response time therefore combined the time it took for the driver to make a decision and perceptual and motor delays directly associated with the decision. For "stay" decisions however, appropriate response time measure could not be extracted from the data because unlike "go" decisions, there are no clues in the recorded data that mark the moment when the "stay" decision was made.

In this paper we focus on left-turn gap acceptance decisions. We therefore did not analyze the trials in which the participants did not evaluate a gap, which was the case if they a) performed right turns; b) went straight through the intersection, or c) did not fully stop before the left turn so the oncoming car did not appear at the intersection; the latter happened in 27 (1.4\%) left turns. Finally, we excluded 10 (0.5\%) "go" decisions in which response time could not be determined because the participant had the gas pedal pressed throughout the trial, and 1 left-turn decision with extreme response time (greater than $2 \mathrm{~s}$ ). This resulted in 1878 analyzed left turns. 


\section{Statistical analysis}

Mixed-effects models were used to analyze the effects of distance and TTA on decision outcome (binomial model) and response time (linear model); the models were implemented using R package lme4. In both models, random effects of participant were included to account for individual differences, with the maximum random effects structure permitting model convergence (see the analysis scripts for details). "Stay" decisions were coded as 0 , "go" decisions as 1 .

\section{Data and code availability}

Data collection code, analysis scripts, and the data are available at https://osf.io/x3ns6/.

\section{Experimental results}

\begin{tabular}{rrrrr}
\hline & Estimate & Std. Error & $\mathrm{z}$ value & $\operatorname{Pr}(>|\mathrm{z}|)$ \\
\hline Intercept & -0.28 & 0.28 & -1 & 0.31 \\
TTA & 1 & 0.11 & 9.1 & $5.7 \times 10^{-20}$ \\
Distance & 1.4 & 0.15 & 9.4 & $5.2 \times 10^{-21}$ \\
\hline
\end{tabular}

Table 2: Results of statistical analysis of the effect of distance and TTA conditions on decision. The binomial mixed-effects model included random slopes of distance and TTA conditions per participant.

\begin{tabular}{rrrrrr}
\hline & Estimate & Std. Error & df & $\mathrm{t}$ value & $\operatorname{Pr}(>|\mathrm{t}|)$ \\
\hline Intercept & -0.72 & 0.033 & 6.8 & -22 & $1.4 \times 10^{-7}$ \\
TTA & 0.028 & 0.0089 & 342 & 3.2 & 0.0017 \\
distance & -0.0052 & 0.01 & 344 & -0.52 & 0.6 \\
\hline
\end{tabular}

Table 3: Results of statistical analysis of the effect of distance and time-to-arrival (TTA) conditions on response time

On average, the participants decided to turn before the arrival of the oncoming car ("go") in $47 \%$ and to wait ("stay") in $53 \%$ of left turns. All trials were included in the decision probability analyses, but only "go" trials were included in the response time analyses.

Replicating existing findings from naturalistic studies of driving (Davis \& Swenson, 2004 Patil \& Pawar, 2014), we found that probability of a decision to go increased with initial time-to-arrival $(z=9.1, p<0.001)$ and initial distance $(z=9.4, p<0.001)$ to the oncoming vehicle (Table 22). The existing literature does not report on timing of gap acceptance; interestingly, we found that response time increased with initial time-to-arrival $(t=4.7, p<0.001)$, but there was no evidence that it was affected by initial distance $(t=-1.4, p=0.16)$ (Table 3).

\section{Evidence accumulation model of gap acceptance decisions}

We hypothesized that the decision-making process of the driver when choosing whether to stay or go is based on noisy integration of sensory evidence. This integration is subject to noise, and is terminated when sufficient evidence is accumulated. Such decision-making processes are often described by the drift-diffusion model (Bogacz, Brown, Moehlis, Holmes, \& Cohen, 2006; Ratcliff \& McKoon, 2008), which we adopt as a basis for our model. However, the complexity of our gap acceptance task poses fundamental challenges for the classical drift-diffusion model and the likes (see online supplementary information at https://osf.io/5nksg/). We address this issue by generalizing the drift-diffusion model in two ways: first, the evidence accumulation rate is determined by a time-varying perceptual evidence, and, second, the amount of evidence that needs to be accumulated decreases with closing time gap (Figure 4). 

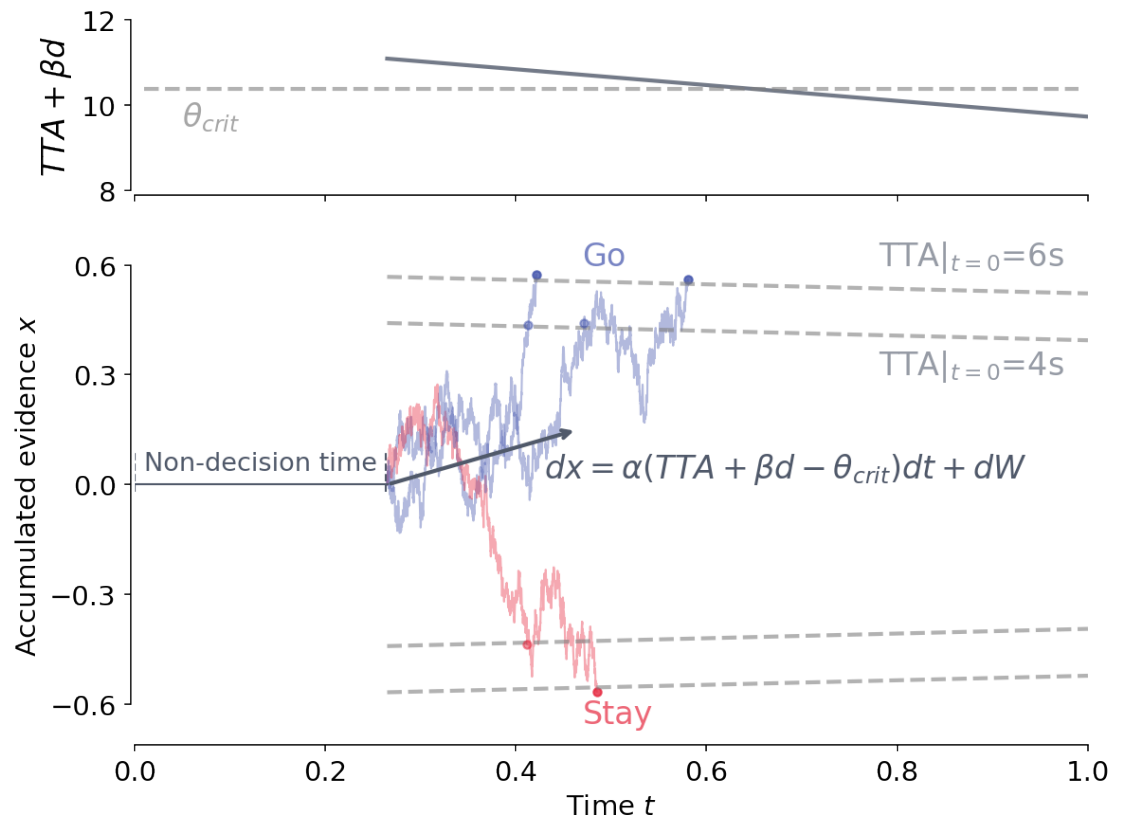

Figure 4: Diagram of the drift-diffusion model of driver's decision making. Relative evidence in favor of going over staying is accumulated until it reaches either a positive or a negative boundary, at which point a "go" or "stay" decision is made, respectively. The rate of accumulation depends on a linear combination of the current values of TTA and $d$ (upper panel). Boundaries for two TTA conditions are plotted for comparison. For each trace, the markers illustrate the moment when the decision is made at the boundaries corresponding to TTA $\left.\right|_{t=0}=4 \mathrm{~s}$ and $6 \mathrm{~s}$. For simplicity, non-decision time is illustrated as a perception delay before the start of evidence accumulation; in our model, non-decision time also accounts for the response delay after the decision is made.

\section{Time-varying perceptual evidence determines accumulation rate}

First, in our left-turn scenario, the incoming evidence dynamically varies over the time course of each decision. In this scenario, the main perceptual quantities driving the drivers' decisions are distance and time-to-arrival (Table 2, see also Davis and Swenson (2004)). However, unlike typical laboratory experiments on decision making, these perceptual quantities systematically and substantially change during several hundreds of milliseconds needed to make a decision. Therefore, the canonical assumption of fixed accumulation rate does not hold in our case. To address this, our model hypothesizes that the accumulation rate dynamically depends on distance and time-to-arrival, and is therefore time-dependent.

Assuming that evidence accumulation is driven by a linear combination of distance and time-to-arrival (Giles et al., 2019), its dynamics can described by the stochastic differential equation

$$
d x=\alpha\left((\mathrm{TTA}+\beta d)-\theta_{\text {crit }}\right) d t+d W,
$$

where $x$ is the decision variable, TTA $=\mathrm{TTA}(t)$ is the time-to-arrival of the oncoming car at time $t, d=d(t)$ is the distance to the oncoming car at time $t$, and $W$ is a stochastic Wiener process. The drift rate parameter $\alpha \geq 0$ quantifies the relative contribution of incoming perceptual information to the accumulated evidence (decisions are made at random if $\alpha=0$ ). Relative weighting of distance information (compared to time-toarrival) is characterized by parameter $\beta$. Parameter $\theta_{\text {crit }}$ determines the "critical" value of TTA $+\beta d$, such that at the time TTA $+\beta d=\theta_{\text {crit }}$, the drift rate changes sign.

\section{Task constraints drive urgency effect via collapsing boundaries}

Second, with the oncoming car approaching the intersection, the window of opportunity for the driver to go closes fast, which inherently creates an urgency effect: When the time-to-arrival of the oncoming vehicle is large, there is no time pressure for the driver to make a decision, and the driver therefore can sample as much 
evidence as needed to make an informed decision. However, when the time-to-arrival is small, the driver is compelled to finalize the decision as fast as possible in order to allow enough time for the turning manoeuvre in case the decision is made to go. Previously, similar time pressure effects in evidence accumulation have been modelled by decision boundaries collapsing over time (Churchland, Kiani, \& Shadlen, 2008. Drugowitsch, Moreno-Bote, Churchland, Shadlen, \& Pouget, 2012). We adopt the same approach in our model, with one important distinction. As the decision urgency in our setup increases with decreasing TTA, we assume the decision boundary to collapse with TTA: the lower the TTA, the less evidence the driver needs to accumulate in order to arrive to a decision.

To capture these considerations, our model assumes that the dynamic accumulation process (1) is terminated when evidence $x$ hits one of the boundaries

$$
b(t)= \pm b_{0} f(\operatorname{TTA}(t))
$$

where $b_{0}$ is the boundary scale parameter, and $f(\cdot)$ is an increasing function of TTA such that

$$
f(\operatorname{TTA}(t)) \approx\left\{\begin{array}{ll}
1 & \text { if } \operatorname{TTA}(t) \gg \tau \\
1 / 2 & \text { if } \operatorname{TTA}(t) \approx \tau . \\
0 & \text { if } \operatorname{TTA}(t) \ll \tau
\end{array} .\right.
$$

For simplicity, we chose a sigmoid function satisfying these conditions

$$
f(\mathrm{TTA})=1 /\left(1+e^{-k(\mathrm{TTA}-\tau)}\right),
$$

where the parameter $k>0$ defines the sensitivity of boundary to time-to-arrival, and $\tau$ is the time-to-arrival at which the boundary is at its baseline value $\left( \pm \frac{1}{2} b_{0}\right)$.

\section{Perception and action delays}

Our model only captures the decision process itself, and does not represent sensory perception and decision execution, and, consequently, any time delays associated with them. These delays are necessarily present in the experimentally measured response times, and are typically modelled by singling out a non-decision component of the overall response time (that is, response time is the sum of decision time and non-decision time) (Ratcliff \& McKoon, 2008. Ratcliff \& Tuerlinckx, 2002). We included a normally distributed non-decision time in the model

$$
t^{\mathrm{ND}} \in \mathcal{N}\left(\mu_{\mathrm{ND}}, \sigma_{\mathrm{ND}}\right)
$$

\section{Model fitting}

In total, our model (1)-(4) has eight free parameters: $\alpha, \beta, \theta_{\text {crit }}, b_{0}, k, \tau, \mu_{\mathrm{ND}}, \sigma_{\mathrm{ND}}$. We implemented the model in pyddm (Shinn, Lam, \& Murray, 2020), and fitted it to the data using differential evolution optimization of the weighted least-sum score (Ratcliff \& Tuerlinckx, 2002).

To assess the explanatory power of the model, we fitted it to the data obtained from each participant individually. In addition, to characterize the typical behavior over all participants, we fitted the model to the group-averaged probabilities and response time distributions. To quantify the group-averaged response time distributions across participants, we used the vincentizing approach (Ratcliff, 1979 Rouder \& Speckman, 2004 Vincent, 1912): based on the individual participants' data, we calculated per-participant RT quantile functions, which were then averaged across participants. The group-averaged cumulative distribution function was then calculated as an inverse of the group-averaged quantile function. The group means of response times and probability of going were calculated as the average of within-participant mean values.

\section{Modeling results}

\section{Evidence accumulation model explains and predicts the experimental results}

The model explained the observed effect of distance and time-to-arrival on probability of a go decision (Figure 5). Not all participants were described equally well, in particular, the behavior of participant 16 posed a problem for the model. However, overall the model exhibited a full range of behaviors observed in the participants, capturing the behavior of participants whose decisions were relatively weakly affected by the 
P1
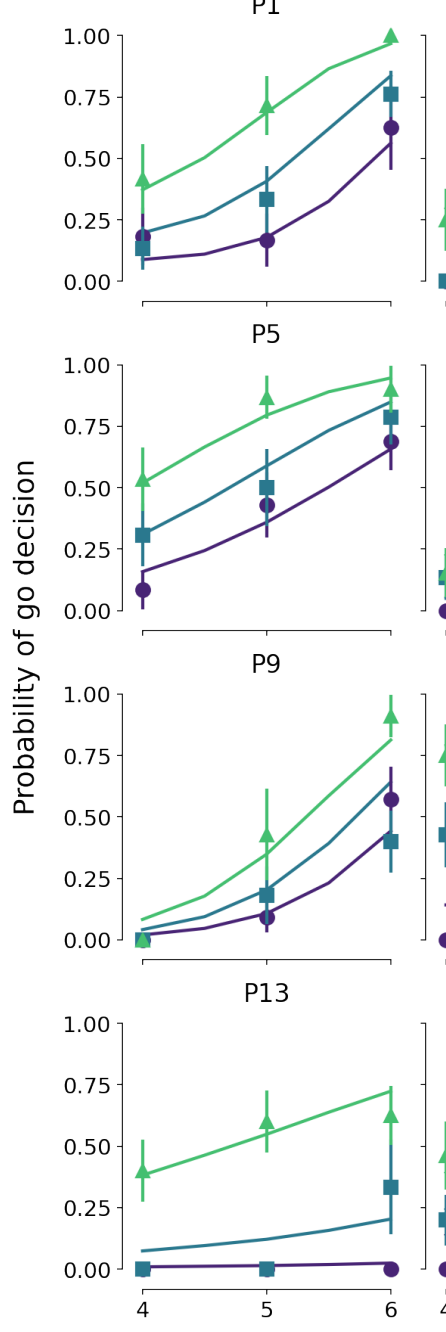

P2

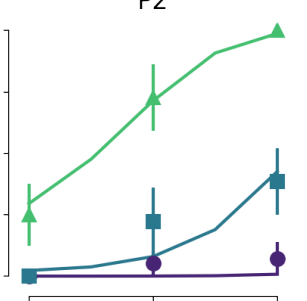

P6

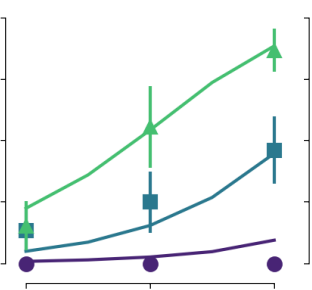

P10

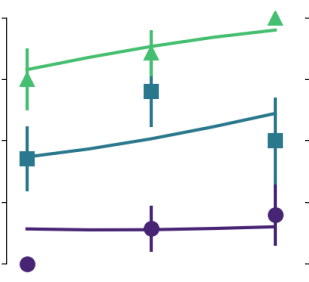

P14

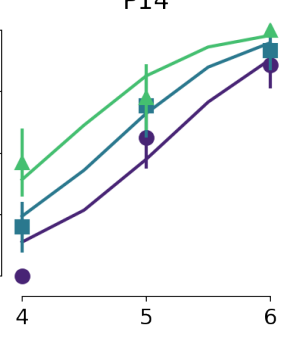

P3

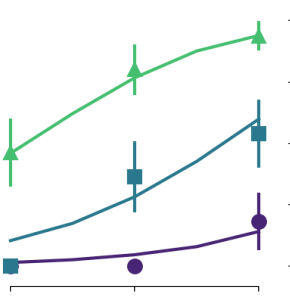

P7

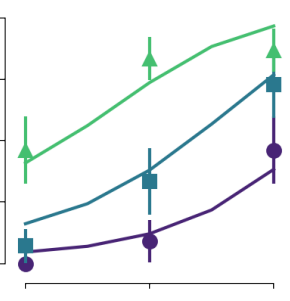

P11
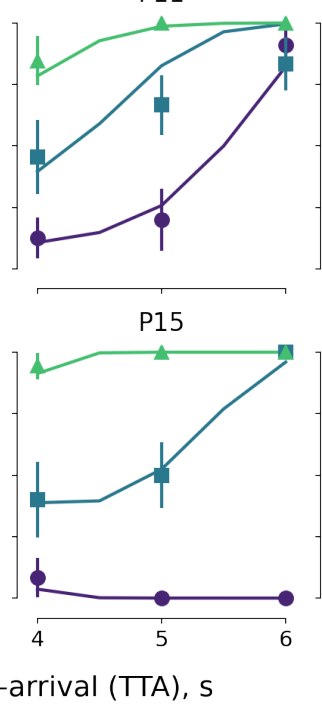

P4

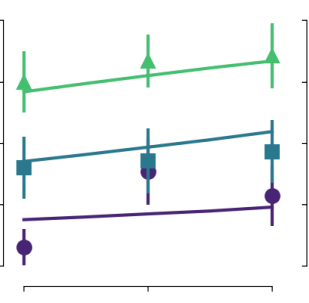

P8
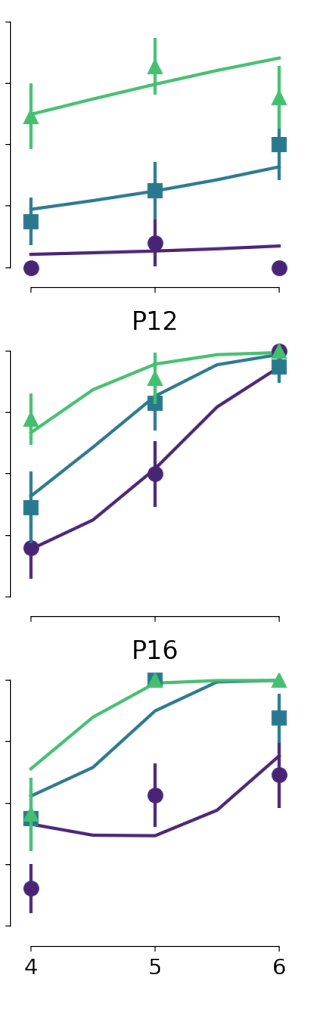

Figure 5: Probability of "go" decision as a function of distance and time-to-arrival of the oncoming car. Individual participants' and group-averaged data (markers) and behavior of the model fitted to the respective datasets (lines). The markers in the 'all participants' panel represent probabilities averaged over withinparticipant mean values. Error bars denote binomial proportion standard error of mean.

distance condition (e.g., participant 14), as well as those who seemed to decide mostly based on the distance and not time-to-arrival (e.g., participants 10 and 13).

Crucially, the model captured the observed response times of all sixteen drivers, as well as the group-averaged response times. Specifically, the model explained the positive relationship between response time and time-toarrival in the group-averaged data (Figure 6). Notably, the model could capture a range of the effect sizes across participants (e.g., participant 1 vs participant 3).

Full response time distributions provide further evidence supporting our model. The model fitted to the group response times generated cumulative distribution functions which followed the group-averaged estimates in eight out of nine conditions (Figure 7). In the remaining condition with the shortest TTA and smallest distance $(\mathrm{TTA}=4 \mathrm{~s}, d=90 \mathrm{~m})$ however, there were not enough "go" responses to reconstruct the full distribution (only 11 response times across 16 participants). The match between the group-averaged distributions and the model in all other conditions however indicates that the model did not simply fit the "go" probability and mean response times, but also captured the full range of response times generated by the participants. 
P1

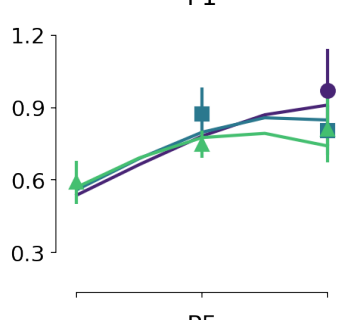

P5

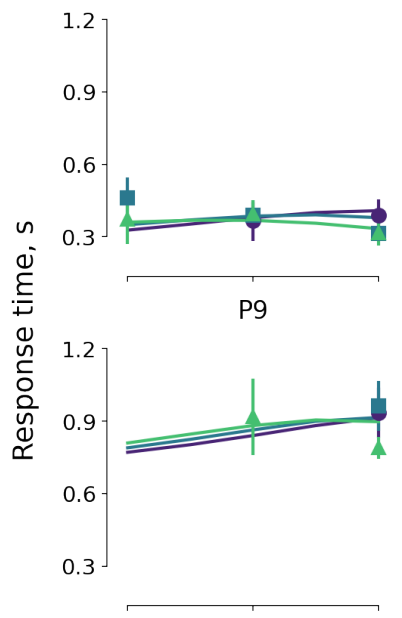

P13

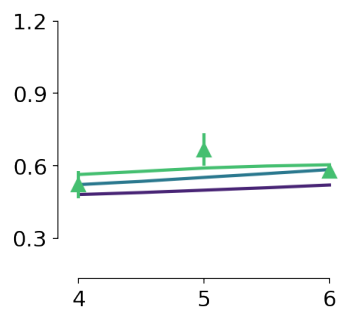

P2

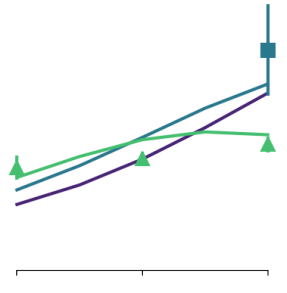

P6

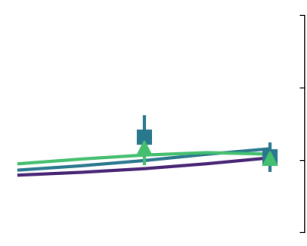

P10

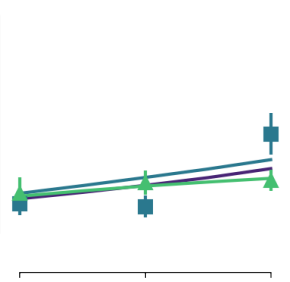

P14

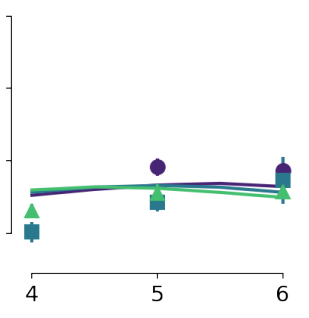

P3

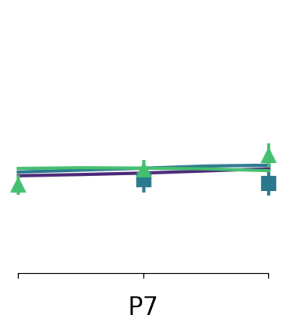

P7

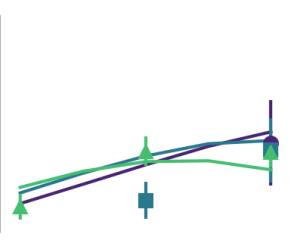

P11

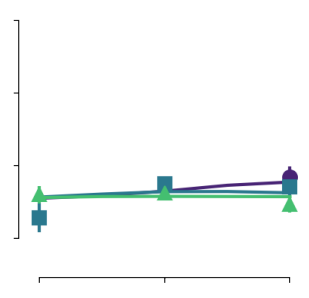

P15

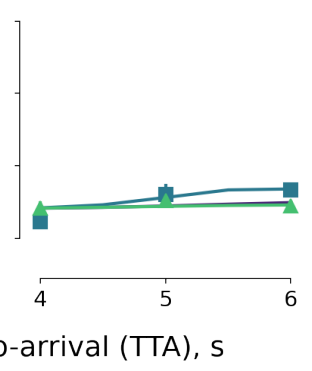

P4
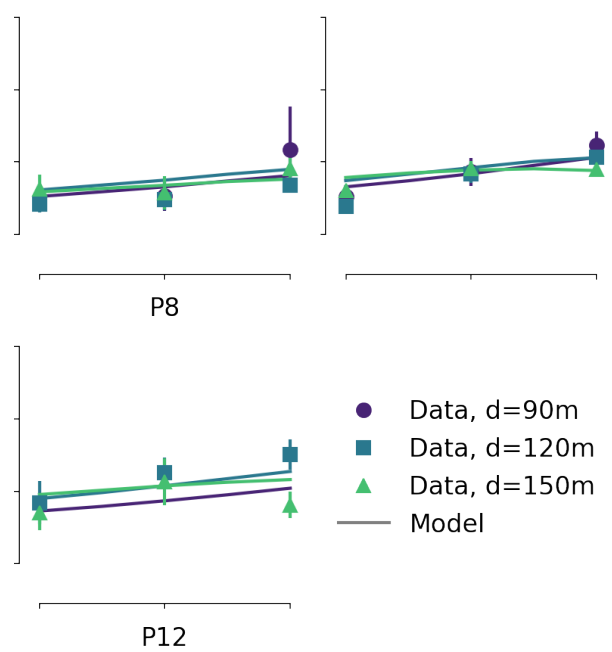

Time-to-arrival (TTA), s

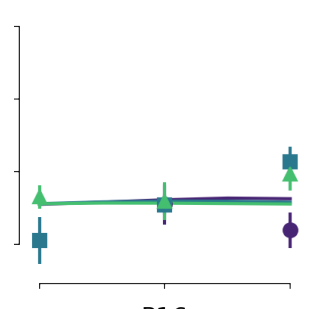

P16

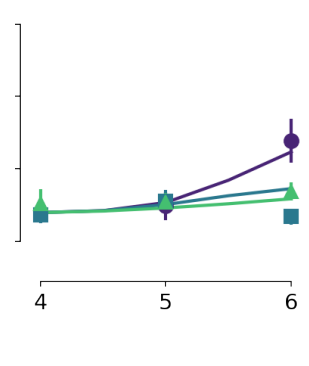

Figure 6: Response time in the "go" decisions as a function of distance and time-to-arrival of the oncoming car. Individual participants' and group-averaged data (markers) and behavior of the model fitted to the respective datasets (lines). The markers in the 'all participants' panel represents response times averaged over within-participant mean values. Response times for conditions with less than three data points are omitted. Error bars indicate standard error of mean.

In all analyses above, the model was fitted to all data available from an individual participant, or the group-averaged data. This allowed us to assess whether the model could explain the observed behavior, but not whether it generalizes beyond the data used for fitting, which would not be the case, for instance, if the model was overfitted.

To investigate how the model generalizes to out-of-sample conditions, and at the same time highlight any potential cases of overfitting, we performed cross-validation of the model using a hold-one-condition-out procedure, focusing on the group-averaged data. For each of the nine (TTA, $d$ ) conditions, we fitted the model to the data from the remaining eight conditions. The fitted parameters were then used to predict the decision outcomes and mean response times in the ninth, held-out condition. This was repeated nine times, for nine held-out combinations of time and distance gaps.

The model predictions in this cross-validation setting still matched the observed decision probabilities and response time (Figure 8), albeit less well than when fitting to full data. Of all conditions, the response times predicted by the model deviated furthest from the data for TTA $=4 \mathrm{~s}, d=150 \mathrm{~m}$. This indicates a certain 


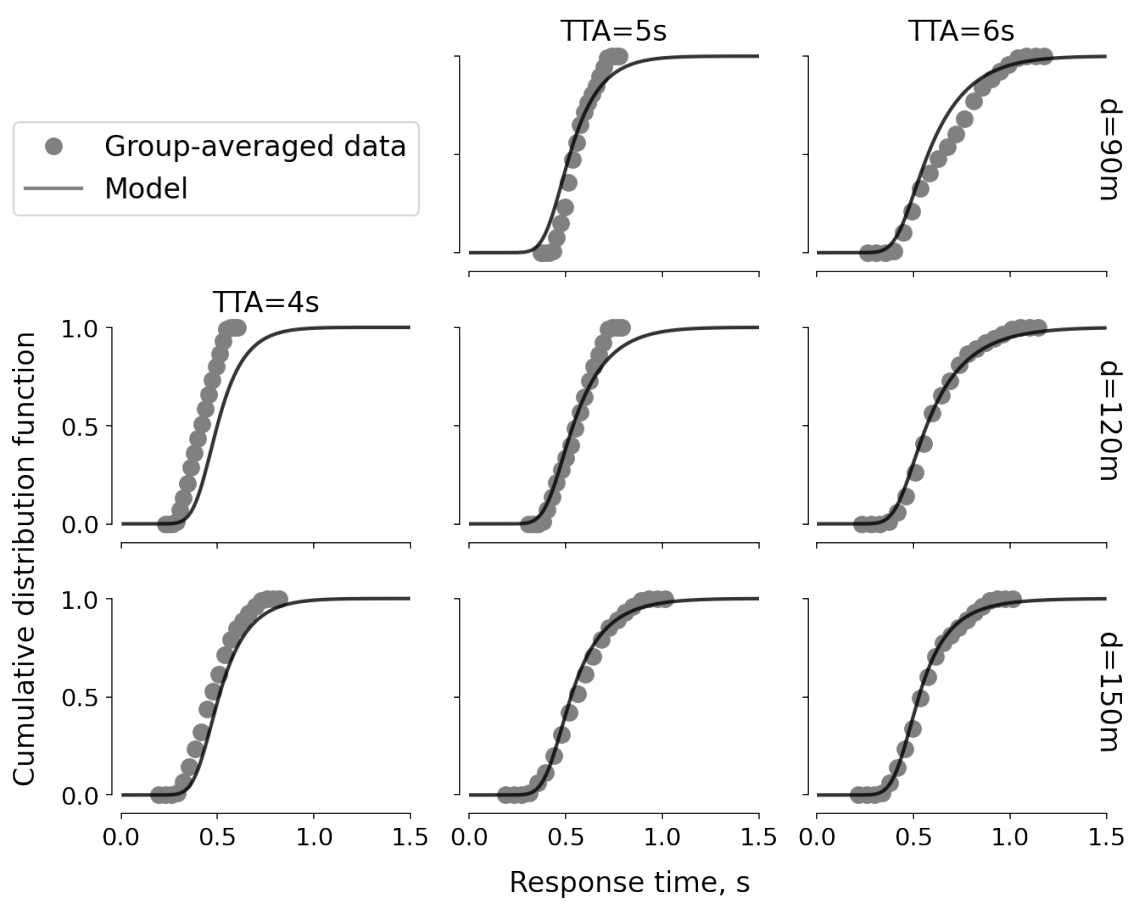

Figure 7: Full distributions of response time in "go" decisions: group-averaged cumulative distribution functions (markers) and distributions generated by the model fitted to the group-averaged response times (solid lines). Distribution for TTA $=4 \mathrm{~s}, d=90 \mathrm{~m}$ is not visualized because all participants together made only 11 "go" decisions in this condition.

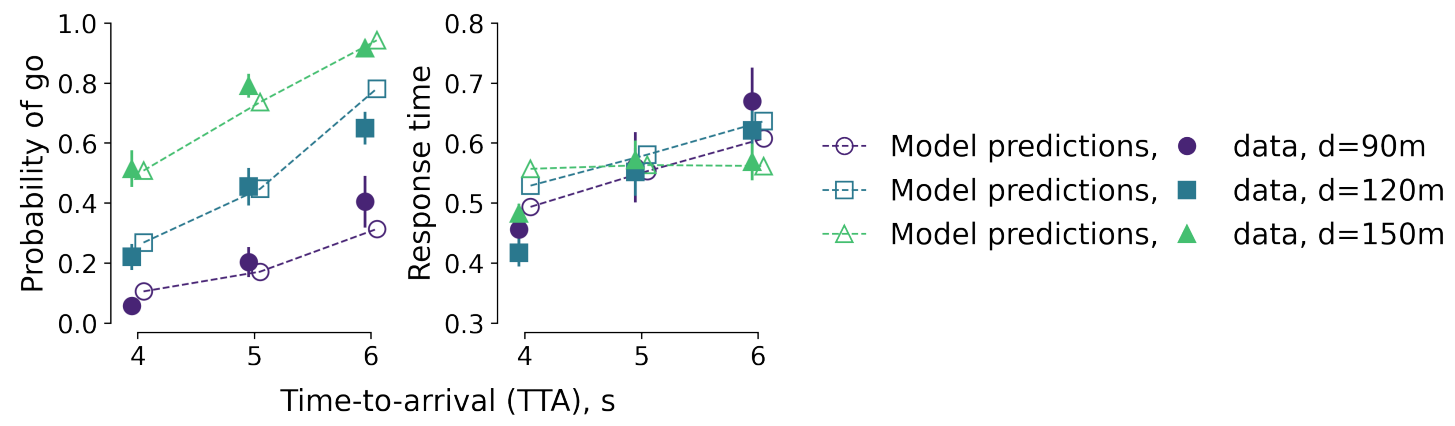

Figure 8: Hold-one-condition-out cross-validation of the model. Filled markers represent the group-averaged data (same as in the "all participants" panels in Figs. 5 and 6). Hollow markers indicate model-generated predictions for the held-out condition. Dashed lines connect the hollow markers for visual clarity and do not represent outputs of the model.

degree of overfitting, possibly highlighting that this condition (yielding in a somewhat extreme oncoming car speed of $37.5 \mathrm{~m} / \mathrm{s}$ ) is close to the edge of the model's feasibility scope. Notably, for other conditions excluding parts of the data from the training set did not substantially affect the simulated model behavior and therefore the match between its predictions and the data. Overall, the cross-validation results suggest that the model's predictions of the "average" participant's behavior can generalize to out-of-sample conditions.

\section{Comparison to simpler evidence accumulation models}

To investigate whether our observations could be explained by simpler evidence accumulations models, we have fitted two simplified versions of our model to the data (see online supplementary information at https://osf.io/5nksg/). The first alternative model had the constant drift rate and constant decision bound; 
the second one included time-varying drift rate but constant decision bound. Both these models could account for the decision outcome data as accurately as the model considered here. However, these models failed to capture the observed effect of time-to-arrival on response time. This suggests that both the time-varying evidence accumulation rate and collapsing decision bounds play essential role in the participants' behavior.

\section{Discussion}

Human drivers make high-stakes decisions on a daily basis; understanding and predicting these decisions can facilitate the design of safe and efficient transportation systems. To develop such an understanding of left-turn decisions, we build upon the classical drift-diffusion model, and extend it with two key notions. First, our model has a variable drift rate linked to the relevant time-varying perceptual quantities - distance and time-to-arrival to the oncoming vehicle - which are dynamically sampled from the environment. Second, the model relies on collapsing decision boundaries, reflecting the impending constraints imposed on the decision execution by the environment. The model explained the observed decision outcomes and response times, as well as substantial individual differences in both. Cross-validation demonstrated that the model not only explained the data, but also generalized to out-of-sample conditions, effectively providing a way to predict human drivers' behavior.

Models of gap acceptance have traditionally focused only on the final outcome of a decision, and the factors affecting it (Davis \& Swenson, 2004 Farah et al., 2009 Hamed et al., 1997. Toledo, 2007). Our work adds to this literature by emphasizing the process of a decision. In practice, process models of traffic decisions yielding predictions of response times can help to optimize safety and traffic flow efficiency (Markkula, Romano, et al., 2018). More fundamentally, modeling the process of drivers' decisions provides an insight into the mechanisms of decision making which are not tapped into when using standard laboratory paradigms.

Our study is not the first to suggest that evidence accumulation can play a role in behavior on the road; models of this nature have been applied to driver steering and braking decisions during basic sensorimotor control of the vehicle (Markkula, Boer, et al., 2018 Ratcliff, 2015 Ratcliff \& Strayer, 2014. Xue et al., 2018), as well as to more complex decisions such as pedestrian road-crossing (Giles et al., 2019 Markkula, Romano, et al., 2018). Some of this research has achieved high methodological rigour, on par with the laboratory research on decision-making, by collecting large number of data points per participant (Ratcliff, 2015 Ratcliff \& Strayer, 2014). To make this possible, these studies used paradigms with speeded response to discrete-onset stimuli, such as brake lights, thus not addressing the types of continuously time-varying sensory information that is crucial in many aspects of driving (Markkula, Engström, Lodin, Bärgman, \& Victor, 2016 Toledo, 2007). Other work has considered time-varying sensory information, but has not been as rigorous in the model-fitting approach, for example absorbing both intra-individual and inter-individual variability into the evidence accumulation noise (Giles et al., 2019 Markkula, Boer, et al., 2018, Xue et al., 2018). The crossing behavior modeling study most similar to ours was that of Giles et al. (2019), who aimed to capture pedestrian crossing decisions. They fitted a number of model candidates to the empirical data, but by own admission they were not able to draw firm conclusions due to limitations in their model-fitting approach. Therefore, to the best of our knowledge, our study is the first to demonstrate methodologically stringent evidence for the value of this type of decision-making model in a driving task with continuously varying sensory evidence.

One of the key notions highlighted by our results is that decisions based on time-varying evidence can be modelled by a drift-diffusion model with the drift rate directly linked to the relevant dynamic perceptual quantities in the environment. This goes beyond the traditional approaches to decision making. These approaches almost ubiquitously focus on the stimuli with the magnitude which is constant throughout the trial, which is naturally modelled by evidence accumulation with constant drift rate (Bogacz et al., 2006, Ratcliff \& McKoon, 2008; Ratcliff, Smith, Brown, \& McKoon, 2016). In laboratory setting, variable-drift evidence accumulation is rarely investigated, mostly as a way of modeling urgency signals (Cisek, Puskas, \& El-Murr, 2009 Murphy, Boonstra, \& Nieuwenhuis, 2016), non-uniform temporal weighting of evidence throughout the trial (Bronfman, Brezis, \& Usher, 2016 Eckhoff, Holmes, Law, Connolly, \& Gold, 2008), and artificially introduced perturbances in sensory evidence (Cheadle et al., 2014 Shinn, Ehrlich, Lee, Murray, \& Seo, 2019). The previous studies on action initiation in intermittent motor control (Markkula, Boer, et al., 2018, Zgonnikov \& Markkula, 2018) and the early evidence accumulation-based models of traffic interactions discussed above (Giles et al., 2019 Markkula, Romano, et al., 2018) highlighted the promise of the variable-drift approach. However, due to complexity of the analyzed tasks these studies could not disentangle the effects of evidence accumulation and other mechanisms at play. Reinforcing these initial results, our study provides conclusive evidence that evidence accumulation with variable drift can explain gap acceptance decisions made based on naturalistic time-varying stimuli. 
Our results also emphasize the crucial role of time pressure induced by the impending oncoming car. The experimental data revealed that response times increased with time-to-arrival in most participants (Figure 6); this goes against predictions of drift-diffusion models with fixed boundaries that imply longer response times for low stimulus intensities. The mechanism of our model which allowed it to capture this experimental observation is decision boundaries collapsing with time-to-arrival. Our scenario is therefore interestingly different from typical speed-accuracy trade-off paradigms in decision-making research, in which time pressures are external to the task being performed (Churchland et al., 2008, Drugowitsch et al., 2012 Shinn et al., 2019). In contrast, in the left-turn decisions, the perceptual information being decided upon (time-to-arrival) simultaneously dictates the urgency. Indeed, the time gap which is closing fast imposes an inherent dynamic constraint on decision execution, which the driver has to take into account when deciding.

Our work goes beyond the decision outcomes and begins to uncover the evidence accumulation mechanisms behind the left-turn decisions, but we consider it to be just the first step towards a comprehensive account of drivers' decision making in gap acceptance. Future work should address the key limitation of our study the lack of a reliable proxy for the duration of the decision process in "stay" decisions. Traditionally, response times for both options are used to constraining the fits of evidence accumulation models. However, in our experiment the measurement of "stay" response times could not be extracted from the vehicle motion data. This could potentially be aided e.g. by asking the participants to press a button as soon as they decide to stay. We however opted for not doing so, because such a button press would not be as automatized a behavior as pushing the gas pedal, which could lead to inflated non-decision time in the "stay" decisions and interfere with the decision making process in "go" decisions. Follow-up studies should develop ecologically valid ways of capturing "stay" response times.

Duration of a decision process provides a useful but coarse-grained measurement of a cognitive process. Going beyond response times, dynamic measures such as movement trajectories can provide researchers with rich data about a cognitive process (Schulte-Mecklenbeck et al., 2017, Zgonnikov, Atiya, O'Hora, Rañó, \& Wong-Lin, 2019). This includes, for instance, information on gradual or abrupt reversal of preference prior to commitment to the final decision (changes-of-mind). Our data indicates that up to $15 \%$ of "go" trials could potentially include a change-of-mind when a driver first accelerates soon after seeing the oncoming car, but then stops short of taking the turn, waiting until the car passes instead. However, within our experimental procedure, such changes-of-mind cannot be reliably distinguished from "rolling" behaviors when the drivers simply inch forward after making the "stay" decision in order to take the turn faster after the oncoming vehicle had passed. This is further complicated in case of changes from "stay" to "go", which cannot be identified based on vehicle motion data alone. Developing a way of reliably identifying changes-of-mind can enable modeling them, which can in turn clarify to what extent they are caused by continued accumulation of new evidence (Fleming, van der Putten, \& Daw, 2018 Resulaj, Kiani, Wolpert, \& Shadlen, 2009) or high level of uncertainty about the initial decision (Atiya, Rañó, Prasad, \& Wong-Lin, 2019 Atiya et al., 2020).

A general limitation of the evidence accumulation models which also extends to our model is the lack of mechanistic explanations of post-decision processes such as decision execution (Evans \& Wagenmakers, 2019). In the context of our task, this means that our model cannot account e.g. for the rate of acceleration applied after the "go" decision is made. One possible approach to extend our model in this direction could be through decision confidence (Kepecs, Uchida, Zariwala, \& Mainen, 2008; Kiani \& Shadlen, 2009): hypothetically, the more confident the driver is in their "go" decision, the stronger they press the gas pedal to execute it. However, this approach requires further experimental investigations to collect per-trial confidence judgments in a non-intrusive manner. Furthermore, it is not clear whether the drift-diffusion model is an adequate framework for capturing decision confidence (Ratcliff et al., 2016 Yeung \& Summerfield, 2012). Future studies should investigate the relation between evidence accumulation and response execution in drivers' decisions both experimentally and computationally.

Our work has potential practical applications for implementing human models in real-time prediction of human behavior in traffic, as well as virtual environments for testing automated vehicles. Most research on real-time human prediction in traffic uses computationally convenient but cognitively implausible models of human behavior, for instance fixed decision probability (Kooij, Flohr, Pool, \& Gavrila, 2019; Thornton, Limonchik, Lewis, Kochenderfer, \& Gerdes, 2019) and utility maximization (Sadigh, Landolfi, Sastry, Seshia, \& Dragan, 2018: Schwarting, Pierson, Alonso-Mora, Karaman, \& Rus, 2019), which is known to diverge substantially from actual human behavior (Kahneman \& Tversky, 1979), including that in driving (Schmidt-Daffy, 2014; Siebinga, Zgonnikov, \& Abbink, 2021). Therefore, having cognitively grounded models of human behavior in traffic interactions can help to generate more accurate and generalizable predictions of road user behavior. Our study provides a step forward here, but needs to be complemented with further work on other scenarios, and translated to real-world contexts with higher variability than in typical controlled studies. 
Taken together, our results suggest that dynamic evidence accumulation underlies left-turn gap acceptance decisions in human drivers. More generally, this study exemplifies how simple cognitive process models can help us to understand human behavior in complex real-world tasks.

\section{Acknowledgments}

This research was funded in part by the UK Engineering and Physical Sciences Research Council (EPSRC) grant EP/S005056/1.

We thank Joris Giltay for the help in configuring the driving simulator, and Catholijn Jonker for helpful feedback on the earlier versions of this manuscript. We thank VectorZero for a free license of the RoadRunner software used to design the road layout for the experiment.

\section{References}

Atiya, N. A. A., Rañó, I., Prasad, G., \& Wong-Lin, K. (2019). A neural circuit model of decision uncertainty and change-of-mind. Nature Communications, 10(1), 2287. doi:10.1038/s41467-019-10316-8

Atiya, N. A. A., Zgonnikov, A., O'Hora, D., Schoemann, M., Scherbaum, S., \& Wong-Lin, K. (2020). Changesof-mind in the absence of new post-decision evidence. PLOS Computational Biology, 16(2), e1007149. doi:10.1371/journal.pcbi.1007149

Bogacz, R., Brown, E., Moehlis, J., Holmes, P., \& Cohen, J. D. (2006). The physics of optimal decision making: A formal analysis of models of performance in two-alternative forced-choice tasks. Psychological Review, 113(4), 700-765. doi:10.1037/0033-295X.113.4.700

Bronfman, Z. Z., Brezis, N., \& Usher, M. (2016). Non-monotonic temporal-weighting indicates a dynamically modulated evidence-integration mechanism. PLoS computational biology, 12(2), e1004667.

Cheadle, S., Wyart, V., Tsetsos, K., Myers, N., De Gardelle, V., Castañón, S. H., \& Summerfield, C. (2014). Adaptive gain control during human perceptual choice. Neuron, 81 (6), 1429-1441.

Churchland, A. K., Kiani, R., \& Shadlen, M. N. (2008). Decision-making with multiple alternatives. Nature Neuroscience, 11(6), 693-702. doi $10.1038 / \mathrm{nn} .2123$

Cisek, P., Puskas, G. A., \& El-Murr, S. (2009). Decisions in changing conditions: The urgency-gating model. Journal of Neuroscience, 29(37), 11560-11571.

Davis, G. A., \& Swenson, T. (2004). Field Study of Gap Acceptance by Left-Turning Drivers. Transportation Research Record, 1899 (1), 71-75. doi:10.3141/1899-09

Dosovitskiy, A., Ros, G., Codevilla, F., Lopez, A., \& Koltun, V. (2017). CARLA: An Open Urban Driving Simulator. arXiv:1711.03938 [cs].

Drugowitsch, J., Moreno-Bote, R., Churchland, A. K., Shadlen, M. N., \& Pouget, A. (2012). The Cost of Accumulating Evidence in Perceptual Decision Making. Journal of Neuroscience, 32(11), 3612-3628. doi:10.1523/JNEUROSCI.4010-11.2012

Eckhoff, P., Holmes, P., Law, C., Connolly, P. M., \& Gold, J. I. (2008). On diffusion processes with variable drift rates as models for decision making during learning. New Journal of Physics, 10(1), 015006. doi:10.1088/1367-2630/10/1/015006

Evans, N. J., \& Wagenmakers, E.-J. (2019). Evidence Accumulation Models: Current Limitations and Future Directions. PsyArXiv. doi:10.31234/osf.io/74df9

Farah, H., Bekhor, S., Polus, A., \& Toledo, T. (2009). A passing gap acceptance model for two-lane rural highways. Transportmetrica, 5(3), 159-172. doi 10.1080/18128600902721899

Fleming, S. M., van der Putten, E. J., \& Daw, N. D. (2018). Neural mediators of changes of mind about perceptual decisions. Nature Neuroscience, 21 (4), 617-624. doi:10.1038/s41593-018-0104-6

Giles, O., Markkula, G., Pekkanen, J., Yokota, N., Matsunaga, N., Merat, N., \& Daimon, T. (2019). At the Zebra Crossing: Modelling Complex Decision Processes with Variable-Drift Diffusion Models. In Proceedings of the 41st Annual Meeting of the Cognitive Science Society (pp. 366-372). Cognitive Science Society.

Gold, J. I., \& Shadlen, M. N. [Michael N.]. (2007). The Neural Basis of Decision Making. Annual Review of Neuroscience, 30(1), 535-574. doi:10.1146/annurev.neuro.29.051605.113038

Hamed, M. M., Easa, S. M., \& Batayneh, R. R. (1997). Disaggregate Gap-Acceptance Model for Unsignalized T-Intersections. Journal of Transportation Engineering, 123(1), 36-42.

Jarecki, J. B., Tan, J. H., \& Jenny, M. A. (2020). A framework for building cognitive process models. Psychonomic Bulletin 83 Review. doi:10.3758/s13423-020-01747-2 
Kahneman, D., \& Tversky, A. (1979). Prospect Theory: An Analysis of Decision under Risk. Econometrica, 47(2), 263-291. doi:10.2307/1914185

Kepecs, A., Uchida, N., Zariwala, H. A., \& Mainen, Z. F. (2008). Neural correlates, computation and behavioural impact of decision confidence. Nature, 455(7210), 227-231. doi:10.1038/nature07200

Kiani, R., \& Shadlen, M. N. [M. N.]. (2009). Representation of Confidence Associated with a Decision by Neurons in the Parietal Cortex. Science, 324(5928), 759-764. doi:10.1126/science.1169405

Kooij, J. F. P., Flohr, F., Pool, E. A. I., \& Gavrila, D. M. (2019). Context-Based Path Prediction for Targets with Switching Dynamics. International Journal of Computer Vision, 127(3), 239-262. doi:10.1007/ s11263-018-1104-4

Markkula, G., Boer, E., Romano, R., \& Merat, N. (2018). Sustained sensorimotor control as intermittent decisions about prediction errors: Computational framework and application to ground vehicle steering. Biological Cybernetics, 112(3), 181-207. doi:10.1007/s00422-017-0743-9

Markkula, G., Engström, J., Lodin, J., Bärgman, J., \& Victor, T. (2016). A farewell to brake reaction times? Kinematics-dependent brake response in naturalistic rear-end emergencies. Accident Analysis $\mathcal{E}$ Prevention, 95, 209-226. doi:10.1016/j.aap.2016.07.007

Markkula, G., Romano, R., Madigan, R., Fox, C. W., Giles, O. T., \& Merat, N. (2018). Models of Human Decision-Making as Tools for Estimating and Optimizing Impacts of Vehicle Automation. Transportation Research Record: Journal of the Transportation Research Board, 2672(37), 153-163. doi:10.1177/ 0361198118792131

Matusz, P. J., Dikker, S., Huth, A. G., \& Perrodin, C. (2019). Are We Ready for Real-world Neuroscience? Journal of Cognitive Neuroscience, 31(3), 327-338. doi:10.1162/jocn_e_01276

Murphy, P. R., Boonstra, E., \& Nieuwenhuis, S. (2016). Global gain modūation generates time-dependent urgency during perceptual choice in humans. Nature communications, 7(1), 1-15.

Patil, G. R., \& Pawar, D. S. (2014). Temporal and Spatial Gap Acceptance for Minor Road at Uncontrolled Intersections in India. Transportation Research Record: Journal of the Transportation Research Board, 2461 (1), 129-136. doi:10.3141/2461-16

Ratcliff, R. (1979). Group reaction time distributions and an analysis of distribution statistics. Psychological Bulletin, 86 (3), 446-461. doi $10.1037 / 0033-2909.86 .3 .446$

Ratcliff, R. (2015). Modeling one-choice and two-choice driving tasks. Attention, Perception, 8 Psychophysics, 77(6), 2134-2144. doi:10.3758/s13414-015-0911-8

Ratcliff, R., \& McKoon, G. (2008). The Diffusion Decision Model: Theory and Data for Two-Choice Decision Tasks. Neural Computation, 20(4), 873-922. doi:10.1162/neco.2008.12-06-420

Ratcliff, R., Smith, P. L., Brown, S. D., \& McKoon, G. (2016). Diffusion Decision Model: Current Issues and History. Trends in Cognitive Sciences, 20(4), 260-281. doi:10.1016/j.tics.2016.01.007

Ratcliff, R., \& Strayer, D. (2014). Modeling simple driving tasks with a one-boundary diffusion model. Psychonomic Bulletin \& Review, 21(3), 577-589. doi:10.3758/s13423-013-0541-x

Ratcliff, R., \& Tuerlinckx, F. (2002). Estimating parameters of the diffusion model: Approaches to dealing with contaminant reaction times and parameter variability. Psychonomic Bulletin 8 Review, $9(3)$, 438-481. doi:10.3758/BF03196302

Resulaj, A., Kiani, R., Wolpert, D. M., \& Shadlen, M. N. (2009). Changes of mind in decision-making. Nature, 461(7261), 263-266. doi:10.1038/nature08275

Rouder, J. N., \& Speckman, P. L. (2004). An evaluation of the Vincentizing method of forming group-level response time distributions. Psychonomic Bulletin \& Review, 11(3), 419-427. doi 10.3758/BF03196589

Sadigh, D., Landolfi, N., Sastry, S. S., Seshia, S. A., \& Dragan, A. D. (2018). Planning for cars that coordinate with people: Leveraging effects on human actions for planning and active information gathering over human internal state. Autonomous Robots, 42(7), 1405-1426. doi:10.1007/s10514-018-9746-1

Schmidt-Daffy, M. (2014). Prospect balancing theory: Bounded rationality of drivers' speed choice. Accident; Analysis and Prevention, 63, 49-64. doi:10.1016/j.aap.2013.10.028

Schulte-Mecklenbeck, M., Johnson, J. G., Böckenholt, U., Goldstein, D. G., Russo, J. E., Sullivan, N. J., \& Willemsen, M. C. (2017). Process-tracing methods in decision making: On growing up in the 70s. Current Directions in Psychological Science, 26(5), 442-450. doi:10.1177/0963721417708229

Schwarting, W., Pierson, A., Alonso-Mora, J., Karaman, S., \& Rus, D. (2019). Social behavior for autonomous vehicles. Proceedings of the National Academy of Sciences, 201820676. doi:10.1073/pnas.1820676116

Shamay-Tsoory, S. G., \& Mendelsohn, A. (2019). Real-Life Neuroscience: An Ecological Approach to Brain and Behavior Research: Perspectives on Psychological Science. doi:10.1177/1745691619856350

Shinn, M., Ehrlich, D., Lee, D., Murray, J. D., \& Seo, H. (2019). Confluence of timing and reward biases in perceptual decision-making dynamics. biorxiv. doi:10.1101/865501

Shinn, M., Lam, N. H., \& Murray, J. D. (2020). A flexible framework for simulating and fitting generalized drift-diffusion models. eLife, 9, e56938. 
Siebinga, O., Zgonnikov, A., \& Abbink, D. (2021). Validating human driver models for interaction-aware automated vehicle controllers: A human factors approach. arXiv:2109.13077 [cs].

Stine, G. M., Zylberberg, A., Ditterich, J., \& Shadlen, M. N. (2020). Differentiating between integration and non-integration strategies in perceptual decision making. eLife, 9, e55365. doi:10.7554/eLife.55365

Thornton, S. M., Limonchik, B., Lewis, F. E., Kochenderfer, M. J., \& Gerdes, J. C. (2019). Toward Closing the Loop on Human Values. IEEE Transactions on Intelligent Vehicles, 4 (3), 437-446. doi:10.1109/ TIV.2019.2919471

Toledo, T. (2007). Driving Behaviour: Models and Challenges. Transport Reviews, 27(1), 65-84. doi: $10.1080 /$ 01441640600823940

Vincent, S. B. (1912). The functions of the vibrissae in the behavior of the white rat... Animal Behavior Monographs, 1(5).

Xue, Q., Markkula, G., Yan, X., \& Merat, N. (2018). Using perceptual cues for brake response to a lead vehicle: Comparing threshold and accumulator models of visual looming. Accident Analysis 83 Prevention, 118, 114-124. doi:10.1016/j.aap.2018.06.006

Yeung, N., \& Summerfield, C. (2012). Metacognition in human decision-making: Confidence and error monitoring. Philosophical Transactions of the Royal Society B: Biological Sciences, 367(1594), 13101321. doi: $10.1098 /$ rstb.2011.0416

Zgonnikov, A., Atiya, N. A. A., O'Hora, D., Rañó, I., \& Wong-Lin, K. (2019). Beyond reach: Do symmetric changes in motor costs affect decision making? A registered report. Judgment and Decision Making, $14(4), 15$.

Zgonnikov, A., \& Markkula, G. (2018). Evidence Accumulation Account of Human Operators' Decisions in Intermittent Control During Inverted Pendulum Balancing. In Proceedings of IEEE International Conference on Systems, Man, and Cybernetics (SMC) (pp. 716-721). IEEE. 